\title{
Fast magnetization switching of Stoner particles: A nonlinear dynamics picture
}

\author{
Z. Z. Sun and X. R. Wang \\ Physics Department, The Hong Kong University of Science and Technology, Clear Water Bay, Hong Kong SAR, China
}

(Received 4 January 2005; published 26 May 2005)

\begin{abstract}
The magnetization reversal of Stoner particles is investigated from the point of view of nonlinear dynamics within the Landau-Lifshitz-Gilbert formulation. The following results are obtained. (1) We clarify that the so-called Stoner-Wohlfarth (SW) limit becomes exact when the damping constant is infinitely large. Under the limit, the magnetization moves along the steepest-energy-descent path. The minimal switching field is the one at which there is only one stable fixed point in the system. (2) For a given magnetic anisotropy, there is a critical value for the damping constant, above which the minimal switching field is the same as that of the SW limit. (3) We illustrate how fixed points and their basins change under a field along different directions. This change explains well why a nonparallel field gives a smaller minimal switching field and a short switching time. (4) The field of a ballistic magnetization reversal should be along a certain direction window in the presence of energy dissipation. The width of the window depends on both the damping constant and magnetic anisotropy. The upper and lower bounds of the direction window increase with the damping constant. The window width oscillates with the damping constant for a given magnetic anisotropy. It is zero for both zero and infinite damping. Thus, the perpendicular field configuration widely employed in the current experiments is not the best one since the damping constant in a real system is far from zero.
\end{abstract}

DOI: $10.1103 /$ PhysRevB.71.174430

PACS number(s): 75.60.Jk, 75.75.+a, 05.45.-a

\section{INTRODUCTION}

Magnetic data storage is one of the important components of modern computers. Data input and output involve switching the magnetization of magnetic storage cells (magnetization reversal). The typical switching time with currently used technology is of the order of a nanosecond. If one wants to have a faster computer (modern electronic computers are working at a clock speed of the order of $\mathrm{GHz}$ ), the conventional magnetization reversal method shall soon (the clock speed is doubled every year in the past) become a bottleneck. Thus fast magnetization switching shall be of great importance for the future development of the high-speed information industry.

The reversal of a magnetization can be achieved in many different ways, and it is a very complicated issue. ${ }^{1}$ For example, in a bulk material, magnetization reversal can go through bucking and curling modes or nucleation and domain formation. The recent advance of technology allows us to fabricate the magnetic nanoparticles that are believed to be useful for high-density information storage. ${ }^{2-5}$ For a magnetic nanoparticle, the magnetic moments of all atoms are normally aligned in the same direction, creating a so-called single magnetic domain. Such a nanoparticle is usually called a Stoner-Wohlfarth or Stoner particle. The understanding of magnetization reversal of a single magnetic domain should be relatively simple in comparison with that in a bulk system, but important in nanotechnologies. ${ }^{6}$

There are two challenging issues about magnetization reversal. One is how to have a short reversal time, and the other is how to make the switching field to be small. The conventional magnetization reversal technique is to apply a magnetic field antiparallel to the initial magnetization. A large enough field can drive the initial state out of local minimum and at the same time make the target state to be the global minimum. Thus the system can roll down to the target state through ringing effect. ${ }^{7-9}$ For the issue of a minimal switching field, the classical result, called the StonerWohlfarth (SW) limit, was given by Stoner and Wohlfarth. ${ }^{10}$

Recently picosecond magnetization switching has been observed in experiments ${ }^{11,12}$ by using pulse magnetic fields. Unlike the conventional method, the magnetic field is applied in a perpendicular direction such that the magnetization undergoes a precession. This approach has also received much theoretical attentions. ${ }^{13-16}$ Numerical investigations ${ }^{13}$ showed that the switching time can be substantially reduced because the ringing effect can be suppressed so that the magnetization will move along a so-called ballistic trajectory. ${ }^{16}$ The precessional magnetization reversal provides not only a shorter time but also a lower switching field (well below the SW limit), as found in early numerical calculations. ${ }^{17}$ In the absence of energy dissipation, precessional magnetization switching can also be investigated analytically. Analytical results for the minimal field were obtained by Porter. ${ }^{18} \mathrm{Re}$ cently Xiao and Niu have also studied the minimal field required in the precessional magnetization switching in a conservative system. ${ }^{19}$ Their results were based on fast switching such that energy dissipation can be neglected and the magnetic system can be regarded as a conservative system. They used the phase plane to reveal the properties of magnetization reversal. It was shown that precessional magnetic switching occurs when localized trajectories in the phase plane become delocalized.

Although it is reasonable to approximate a Stoner particle as a conservative system in a short time if the damping (dissipation) during its dynamical motion is small, a real magnetic particle is not a truly conservative system when the damping is large or if one is interested in the long-time behave. Dissipation should be taken into account and a Stoner particle should be treated as a nonconservative system. In this paper we reexamine magnetization reversal as a nonlinear dynamical system in the presence of dissipation. The 

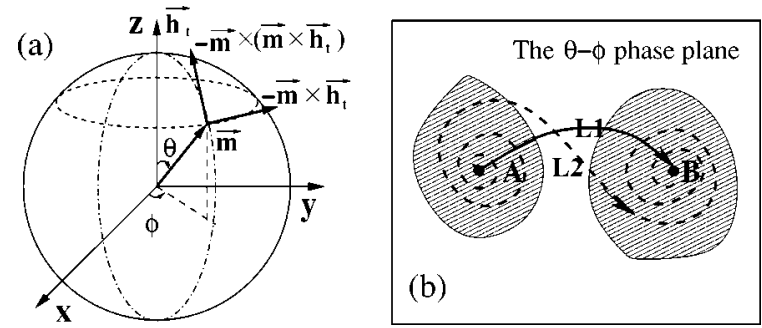

FIG. 1. (a) The magnetization $\vec{m}$ can be uniquely determined by angles $\theta$ and $\phi$. The $z$ axis is assumed to be along the total magnetic field $\vec{h}_{t},-\vec{m} \times \vec{h}_{t}$ determines the precession direction, and $-\vec{m} \times\left(\vec{m} \times \vec{h}_{t}\right)$ decides the dissipation direction. (b) The $\theta-\phi$ phase plane for the magnetization of a Stoner particle. Point $A$ and point $B$ represent the initial and target states, respectively. Two shadowed areas denote schematically basins of two stable fixed points $A$ and $B$. The solid curve $L 1$ and dashed curve $L 2$ illustrate two different phase flows connected to $A$ and $B$.

magnetic dynamics of a single-domain magnetic particle can be described by the evolution trajectories in the phase plane. One can use the general concepts of nonlinear dynamics not only to understand all results from previous studies, but also to see the validity conditions of some of these results such as the SW limit. The paper is organized as follows. In Sec. II we first reformulate magnetization reversal in terms of nonlinear dynamics concepts, such as attractors and phase flow. Previous results are reinterpreted in such language. The Landau-Lifshitz-Gilbert (LLG) equation is introduced. Our main results are presented in Sec. III. The conditions under which the SW limit is valid are given. For a given magnetic anisotropy, we shall show that there is a critical damping constant above which the minimal switching field is the same as that of the SW limit. The reason and meaning of this critical damping constant are also given. The change of fixed points and their basins under a magnetic field is investigated. We show that the field corresponding to ballistic reversal changes from the perpendicular direction for a conservative system to a direction window in the presence of energy dissipation. The conclusion is given in Sec. IV.

\section{NONLINEAR DYNAMICS PICTURE OF MAGNETIZATION REVERSAL}

\section{A. Attractors, phase flow, and magnetization reversal}

Previous results on the magnetization reversal of Stoner particles can be conveniently described in the terminology of nonlinear dynamics. The phase space related to the magnetization is a two-dimensional (2D) plane because all atomic magnetic moments are aligned in the same direction and the magnetization can rotate under an external and/or an internal effective magnetic field that can exert a torque on the spin. The polar angle $\theta$ and the azimuthal angle $\phi$, shown in Fig. 1(a), can fully determine a magnetization $\vec{m}$. In the $\theta-\phi$ plane, each point corresponds to a particular state of the magnetization. A state will in general evolve into new states due to its dynamics. Its motion can be described by a trajectory in the phase plane, called phase flow. The phase flow for a dissipative system ends for a few types of destiny (attractors), including fixed points, limit cycles, or strange attractors. They correspond to stable states, periodic, aperiodic, and chaotic motions. ${ }^{20}$ In a $2 \mathrm{D}$ phase plane, however, the strange attractor solution is not allowed.

The only attractor related to the magnetization reversal of Stoner particles is fixed points. The magnetization reversal problem is as follows: Before applying an external magnetic field, there are two stable fixed points [denoted by $A$ and $B$ in Fig. 1(b)], corresponding to magnetizations-say $\vec{m}_{0}$ (point $A$ ) and $-\vec{m}_{0}$ (point $B$ ) - along its easy axis. The phase plane can be divided into two parts, called basins of attractors. One is around $A$ and the other around $B$, denoted by shadowed areas in Fig. 1(b). The system in basin $A(B)$ will end up at state $A(B)$. Initially, the magnetization is $\vec{m}_{0}$, and the goal is to apply a small external field to switch the magnetization to $-\vec{m}_{0}$ fast.

\section{B. Damping and nondamping magnetization reversals}

The conventional magnetization switching is based on a damping mechanism through, typically, spin-lattice relaxation. From the point of view of nonlinear dynamics, the idea behind the method is to construct the external magnetic field in such a way that the target state is the only stable fixed point. In another word, basin $A$ [Fig. 1(b)] is reduced to zero and the whole (except probably a few isolated points) phase plane is the target-state basin (basin $B$ ). The minimal reversal field (SW limit) is the one at which basin $A$ shrinks to a point. Since the initial and target states have a very large energy difference, the extra energy must be dumped into the lattice during a spiral motion before the system reaches the final state. The system first spirals out of $A$ and then spirals toward $B$, denoted by phase flow $L 2$ in Fig. 1(b). This spiral motion is often referred ${ }^{16}$ to as the ringing effect. The reversal time is largely determined by the effectiveness of energy dissipation.

On the other hand, the target state does not need to be the only fixed point in the recent fast magnetization switching. In fact, it does not even need to be a fixed point. In precessional magnetization reversal, one applies a short magnetic field pulse such that both initial and final states are not fixed points, and system will start to flow in the phase plane. In order to switch the magnetization, one needs to let the system to reach the basin of the final state (basin $B$ ) such that the system will flow to the target state after the pulse field is switched off. Ideally, one wants both initial and target states on its precessional path. This is a nondamping method, and the reversal time does not rely on the spin relaxation time. There are several ways with different control precisions to move the system to the desired state. One way is to apply a perpendicular pulse field to "kick" the system to basin $B$. In comparison with the conventional method, the spiral motion out of the initial state is replaced by a ballistic ${ }^{16}$ motion. However, the system relies on the ringing effect to reach the final state. It was shown ${ }^{13}$ that the switching time can be reduced substantially, but it is still hundreds of picoseconds for a normal magnetic particle due to the ringing effect in the last stage of magnetization reversal. 
The proposal of Xiao and $\mathrm{Niu}^{19}$ is a fully ballistic magnetization reversal scheme. Neglecting the energy dissipation during the motion of a Stoner particle in a magnetic field, the magnetization must move on an equal potential line. Thus the idea is to apply an external magnetic field with a proper strength and in the right direction such that both the initial and target states have the same energy and are on the same phase flow trajectory as schematically illustrated by the solid line $L 1$ connected points $A$ and $B$ with an arrow in Fig. 1(b). Without damping, there is no extra energy to dissipate in this new approach; thus, the system moves from the initial state to the final one in a ballistic way instead of in a ringing mode. The typical time for a precession of $180^{\circ}$ in a field of teslas is of the order of picoseconds for the usual magnetic materials, so a picosecond magnetic field pulse is required in this method. As soon as the system arrives at the target state, one needs to switch off the external field, so the target state becomes a fixed point of the system again. This approach thus requires precise control of the pulse duration.

From the computational point of view, the magnetization reversal time can be evaluated as soon as the phase flow connected the initial and target states is found. The reversal time is given by the length $d l$ of phase flow line divided by the phase velocity $\sqrt{\dot{\theta}^{2}+\dot{\phi}^{2}}$ which is determined by the system dynamics,

$$
t=\int_{A}^{B} \frac{d l(\theta, \phi)}{\sqrt{\dot{\theta}^{2}+\dot{\phi}^{2}}} .
$$

In the language of nonlinear dynamics, an external field modifies the dynamics by changing the phase velocity field. This velocity field is in general a continuous function of the external field. A phase flow between the initial and target states could only have been set up when the external field is strong enough because the initial and final states are two stable fixed points with equally large basins at the beginning. The minimal switching field is the critical one at which such a flow is created.

\section{Landau-Lifshitz-Gilbert equation}

The magnetization dynamics of a Stoner particle is governed by the LLG equation ${ }^{21}$

$$
\frac{d \vec{M}}{d t^{\prime}}=-|\gamma| \vec{M} \times \vec{H}_{t}+\frac{\alpha}{M_{s}} \vec{M} \times \frac{d \vec{M}}{d t^{\prime}},
$$

which can also be written as

$$
\left(1+\alpha^{2}\right) \frac{d \vec{M}}{d t^{\prime}}=-|\gamma| \vec{M} \times \vec{H}_{t}-\frac{\alpha|\gamma|}{M_{s}} \vec{M} \times\left(\vec{M} \times \vec{H}_{t}\right) .
$$

Here $|\gamma|=2.21 \times 10^{5}(\mathrm{rad} / \mathrm{s}) /(\mathrm{A} / \mathrm{m})$ is the gyromagnetic ratio, $M_{s}$ is the saturated magnetization of the particle, and $\alpha$ is the phenomenological dimensionless damping constant. The typical experimental values of $\alpha$ (Ref. 11) range from 0.037 to 0.22 for different Co films. The equation describes the motion of the magnetization vector $\vec{M}$ under an applied magnetic field $\vec{H}$ in the presence of an arbitrary magnetic aniso- tropy energy density function $W(\vec{M}, \vec{H})$. The total field $\vec{H}_{t}=-\nabla_{\vec{M}} W(\vec{M}, \vec{H}) / \mu_{0}=\vec{H}_{\text {eff }}+\vec{H}$, where $\vec{H}_{\text {eff }}$ denotes the internal effective field due to the magnetic anisotropy. $\mu_{0}=4 \pi \times 10^{-7} \mathrm{~N} / \mathrm{A}^{2}$ is the vacuum magnetic permeability.

It is convenient to write the LLG equation in a dimensionless form by defining $\vec{m} \equiv \vec{M} / M_{s}$, the scaled field $\vec{h}_{t} \equiv \vec{H}_{t} / M_{s}=\vec{h}_{e f f}+\vec{h}$, the scaled time $t \equiv t^{\prime} /\left(|\gamma| M_{s}\right)$, and $w(\vec{m}, \vec{h}) \equiv W(\vec{M}, \vec{H}) /\left(\mu_{0} M_{s}^{2}\right)$. The equation for $\vec{m}$ becomes

$$
\left(1+\alpha^{2}\right) \frac{d \vec{m}}{d t}=-\vec{m} \times \vec{h}_{t}-\alpha \vec{m} \times\left(\vec{m} \times \vec{h}_{t}\right) .
$$

As shown in Fig. 1(a), the first term on the right-hand side of Eq. (3) describes the precession motion around the total field and the second term decides the dissipation direction toward the total field. In our analysis, we always use parameters of Co as our references. For Co film, ${ }^{11} M_{s}=1.36 \times 10^{6} \mathrm{~A} / \mathrm{m}$; thus, the time unit is approximately $3.33 \mathrm{ps}$ and the energy unit is $2.32 \times 10^{6} \mathrm{~J} / \mathrm{m}^{3}$.

In terms of $\theta-\phi$, the dynamics of the magnetization is determined by the following nonconservative twodimensional nonlinear autonomous dynamical equations ${ }^{16}$ :

$$
\begin{gathered}
\left(1+\alpha^{2}\right) \dot{\theta}=-\alpha \frac{\partial w}{\partial \theta}-\frac{1}{\sin \theta} \frac{\partial w}{\partial \phi}, \\
\left(1+\alpha^{2}\right) \dot{\phi}=\frac{1}{\sin \theta} \frac{\partial w}{\partial \theta}-\frac{\alpha}{\sin ^{2} \theta} \frac{\partial w}{\partial \phi} .
\end{gathered}
$$

Different particles are characterized by different magnetic anisotropic energy density functions $w(\vec{m}, \vec{h})$. In our analysis, we always assume it to be uniaxial with its easy axis along the $x$ direction. Due to the rotation symmetry around the easy axis, the applied field can be chosen in the $x-z$ plane without losing generality. The general form of $w(\vec{m}, \vec{h})$ can be written as

$$
w(\vec{m}, \vec{h})=f\left(m_{x}\right)-m_{x} h_{x}-m_{z} h_{z},
$$

where $h_{x}$ and $h_{z}$ are the applied magnetic field along the $x$ and $z$ axes, respectively.

This naive-looking nonlinear dynamical equation does not have exact solutions yet. Analytical results can only be obtained in some special cases. For example, analytical solutions were found ${ }^{22,23}$ in the absence of the internal effective field $\left(\vec{h}_{e f f}=0\right)$. The analytical analysis can also be done if there is no energy dissipation as what is shown in Refs. 18,19 . In general, one can use the fourth-order Runge-Kutta method to study the system numerically. In this study, we will use the following $f\left(m_{x}\right)$ in Eq. (5) with different $k_{2}$ and $k_{4}$, whenever we need to use numerical results to illustrate our understandings:

$$
f\left(m_{x}\right)=-\frac{1}{2} k_{2} m_{x}^{2}-\frac{1}{4} k_{4} m_{x}^{4} .
$$

Here $k_{2}, k_{4}$, accounting for the strength of the anisotropy, are positive numbers. In the next section, we shall present our main findings. 


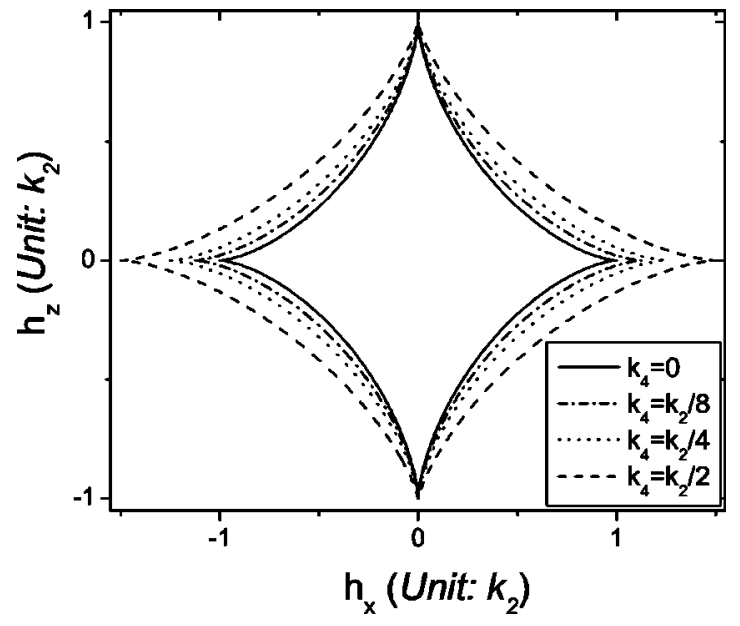

FIG. 2. The SW limit for various choices of $k_{2}, k_{4}$. Solid curve: $k_{4}=0$. Dash-dotted curve: $k_{4}=k_{2} / 8$. Dotted curve: $k_{4}=k_{2} / 4$. Dashed curve: $k_{4}=k_{2} / 2$.

\section{RESULTS AND DISCUSSIONS}

\section{A. Exactness of the SW limit at infinitely large dissipation}

The conventional method is based on the damping mechanism. Its classical result is the so-called SW limit. For uniaxial model with the easy axis along the $x$ axis and magnetic field in the $x z$ plane, the SW limit is obtained by assuming that the magnetization moves in the $x z$ plane during its reversal. The minimal switching field is given by ${ }^{10}$

$$
\begin{aligned}
& \frac{d w}{d m_{x}}=0, \\
& \frac{d^{2} w}{d m_{x}^{2}}=0,
\end{aligned}
$$

with $m_{x}^{2}+m_{z}^{2}=1$. For a widely studied case of $k_{2} \neq 0$ and $k_{4}=0$, the SW limit ${ }^{10}$ is

$$
\left(h_{x} / k_{2}\right)^{2 / 3}+\left(h_{z} / k_{2}\right)^{2 / 3}=1,
$$

corresponding to the solid line in Fig. 2. The SW limits for various choices of $k_{4}$ are also plotted in Fig. 2.

The original SW limit was derived in the static case. ${ }^{10}$ As shown in the dynamical equation (3), the first term on the right-hand side will lift the magnetization away from the $x z$-plane. Thus the assumption of the SW limit that the magnetization moves in the $x z$ plane is only true when this term can be neglected. This will happen when the damping constant becomes infinite $(\alpha \rightarrow \infty)$. In this case, the magnetization will move toward the total field as denoted by the large circle passing through the north-south poles in Fig. 1(a). This is the steepest-energy-descent path for the magnetization. Thus, the minimal switching field of the SW limit corresponds to the one at which there is only one minimum in the energy landscape.

\section{B. Critical value of the damping constant}

In a realistic system, as the damping constant is not infinitely large, the magnetization does not need to move along the steepest-energy-descent path. As a result, a system may still move from the initial state to the local minimum located near the target state even when the external field is smaller than the SW limit. So after the external field is removed, the system will move toward the target state through a ringing mode, achieving magnetization switching. As was shown in many previous studies, ${ }^{17,18}$ minimal switching field can be smaller than the SW limit. Numerical calculations ${ }^{17}$ show that when the damping constant $\alpha<1$, magnetization switching can occur well below the SW limit. While $\alpha \geqslant 1$, the minimal switching field is the SW limit. Thus, it implies that a critical $\alpha_{c}$ exists, above which the minimal switching field is given by the SW limit. In Ref. 17, $\alpha_{c}=1$. To the best of our knowledge, there is no clear understanding of this result. In fact, previous studies ${ }^{17}$ seem to suggest that $\alpha_{c}=1$ is very special. We want to show that there is indeed a critical damping constant for a given magnetic anisotropy. But this critical value can be different for different anisotropy, and $\alpha=1$ is not special. We shall provide an explanation for $\alpha_{c}$.

In order to understand the origin of the critical $\alpha$, let us consider the energy landscape under different external fields. As we mentioned in the previous section, there is only one stable fixed point when $h>h_{S W}$. Asymptotically, the system shall always end up at the fixed point for any nonzero damping. Thus, if one switches off the field after it reaches the fixed point, the system will surely move to the target state (state $B$ ). There is also a $h_{1}<h_{S W}$ at which the energy of system at the initial state equals that at the saddle point between two stable fixed points. Thus there is no way to switch the magnetization when $h<h_{1}$ because the energy of the initial state $(A)$ is too small to overcome the potential barrier between the initial and final states. $h_{1}$ can be determined from the equations

$$
\begin{gathered}
\frac{d w}{d m_{x}}=0, \\
w\left(m_{x}\right)=w_{A},
\end{gathered}
$$

where $w_{A}$ is the energy at the initial state $\left(m_{x}=1\right)$. For a field $h$ between $h_{1}$ and $h_{S W}, h_{1}<h<h_{S W}$, there exist two stable fixed points with a saddle point in between. Furthermore, the energy of the initial state is higher than that of the saddle point. Figure 3 is a schematic $3 \mathrm{D}$ plot of the energy landscape for the case of $h_{1}<h<h_{S W}$. Point $A$ denotes the initial state, whose energy is supposed to be higher than that of the saddle point (SP) between two local minima. In this case, the flow starting from $A$ will finally evolve into either of two fixed points, depending on the value of $\alpha$. When $\alpha$ is infinity, the system will evolve into the minimum near the initial state along the steepest-descent path, as shown by line $R 1$. For the opposite extreme of zero damping $(\alpha=0)$, the system will move along an equal potential contour (line $R 4$ ) surrounding the two minima (fixed points), as investigated in Ref. 19. For small $\alpha$, the magnetization can make many turns around the two local minima before it falls into either one. So there is a special $\alpha=\alpha_{i}$ with which the system just touches the SP when it rolls down from $A$, denoted by dotted line $R 3$. For $\alpha>\alpha_{i}$, the energy damping is too strong for the system to 


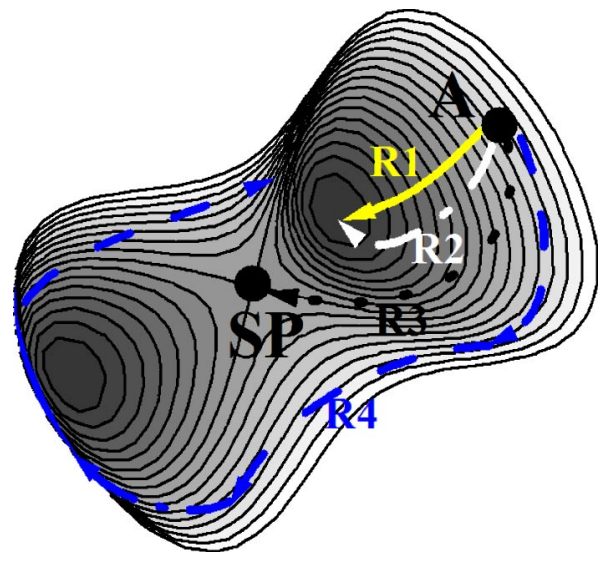

FIG. 3. (Color online) The schematic 3D energy landscape plot of the case $h_{1}<h<h_{S W}$. Point $A$ denotes the initial state, whose energy is supposed to be higher than that of the saddle point (SP). Lines $R 1, R 2, R 3$, and $R 4$ show schematically four typical evolution trajectories for $\alpha=\infty,>\alpha_{i}, \alpha_{i}$, and 0 , respectively.

"climb" over the saddle point, denoted by line $R 2$. The value $\alpha_{i}$ depends obviously on the magnetic field, and the critical damping constant $\alpha_{c}$ is the value of $\alpha_{i}$ at $h=h_{S W}$.

One may also understand the result from Fig. 4 of trajectories of various $\alpha$ in the energy contour plot at $h=h_{S W}$ along $135^{\circ}$ to the $+x$ axis. The result in the figure is for the uniaxial model with $k_{2}=2$ and $k_{4}=0$. As mentioned earlier, the saddle point and one minimum merge together at $h_{S W}$ to form an inflection point denoted by $T$ in Fig. 4. It is clear that all trajectories with $\alpha>\alpha_{c}$ passing through the saddle-inflection point while all those with $\alpha<\alpha_{c}$ do not. One may notice that all curves of $\alpha>\alpha_{c}$ do not move after they reach point $T$ because $T$ is a saddle point. But any small fluctuation will result in the system to leave $T$ and to end up in FP.

In order to demonstrate the correctness of our reasoning for the existence of $\alpha_{c}$ and that the value of the critical damping constant varies with the magnetic anisotropy, we carried out numerical calculations on the uniaxial magnetic

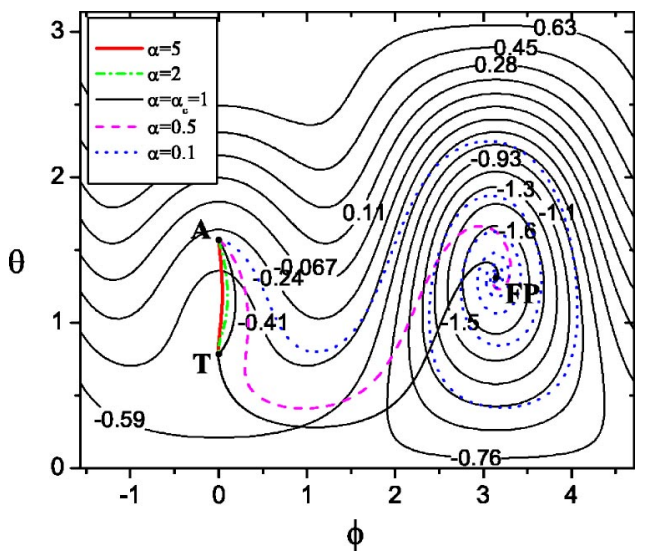

FIG. 4. (Color online) The contour plot of $w(\phi, \theta)$ at $h=h_{S W}$ for the uniaxial magnetic anisotropy model with $k_{2}=2$ and $k_{4}=0$. The field is along $3 \pi / 4$ to the $+x$ axis. Point $A$ is the initial point. FP denotes the stable fixed point. $T$ denotes the inflection point. All flow trajectories of $\alpha \geqslant \alpha_{c}$ touch $T$ while those with $\alpha<\alpha_{c}$ do not.

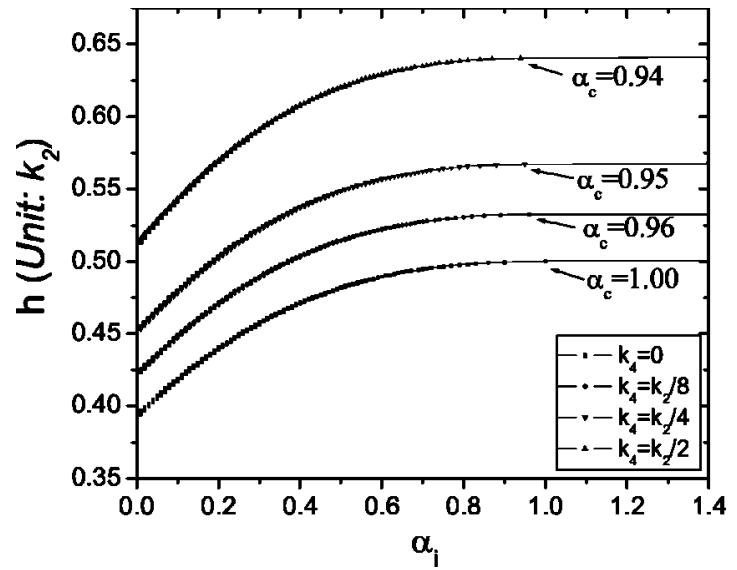

FIG. 5. The minimal switching field versus damping constant $\alpha$. The field is along $3 \pi / 4$ to the $+x$ axis. Square: $k_{4} / k_{2}=0$. Circle: $k_{4} / k_{2}=1 / 8$. Down-triangle: $k_{4} / k_{2}=1 / 4$, Up-triangle: $k_{4} / k_{2}=1 / 2$. Smooth connection curves are to guide the eye.

anisotropy model with different ratios of $k_{4} / k_{2}$. Figure 5 is the $\alpha$ dependence of the minimal switching field for $k_{4} / k_{2}=0,1 / 8,1 / 4,1 / 2$, respectively. Indeed, all curves (dependent only on the ratio of $\left.k_{4} / k_{2}\right)$ saturate to their corresponding SW-limit values $h_{S W}$ after $\alpha$ is greater than certain values, the critical damping constants $\alpha_{c}$. Furthermore, $\alpha_{c}$ is different for different $k_{4}$, varying from $\alpha_{c}=1$ for $k_{4}=0$ to $\alpha_{c}=0.94$ for $k_{4}=k_{2} / 2$. Thus, $\alpha_{c}=1$ is not special at all.

\section{Changes of the basins under an external field}

We would like to investigate numerically how fixed points and their basins change under a field along different directions. In the following calculation, $k_{2}=2, k_{4}=0$, and $\alpha=0.1$ are used. In the absence of an external magnetic field, there are two fixed points at $(\theta=\pi / 2, \phi=0)$ and $(\theta=\pi / 2, \phi=\pi)$, respectively. They are also the initial state $(A)$ and the target state $(B)$. Depending on the direction and strength of an applied field, two fixed points, FP1 and FP2, may move away from $A$ and $B$. The numerical procedures are as follows: Divide the $\theta-\phi$ plane $[\theta \in(0, \pi)$ and $\phi \in(-\pi / 2,3 \pi / 2)]$ into $100 \times 50$ meshes. Each mesh site represents one particular state. Starting from an arbitrary state, the state belongs to the basin of FP1 (FP2) if the system evolves into fixed point FP1 (FP2) after a long enough time. Basin FP1 (FP2) is colored white (grey). Figures 6-8 are the basins with different external fields.

\section{Field along the easy axis}

Figure 6(a) is the basin without an external field. The basins divide the phase plane into two equal-area parts with separatrix lines of $\phi=\pi / 2$ and $3 \pi / 2$. When a field parallel to the easy axis is applied, points $A$ and $B$ are always two fixed points of the system. However, the areas of their basins vary. Figures 6(b)-6(d) show how the area of basin $A$ shrinks while that of $B$ expands as the field increases. When the field reaches the SW limit [Fig. 6(d)], the area of basin $A$ shrinks to zero. Thus $B$ becomes the only stable fixed point and its 


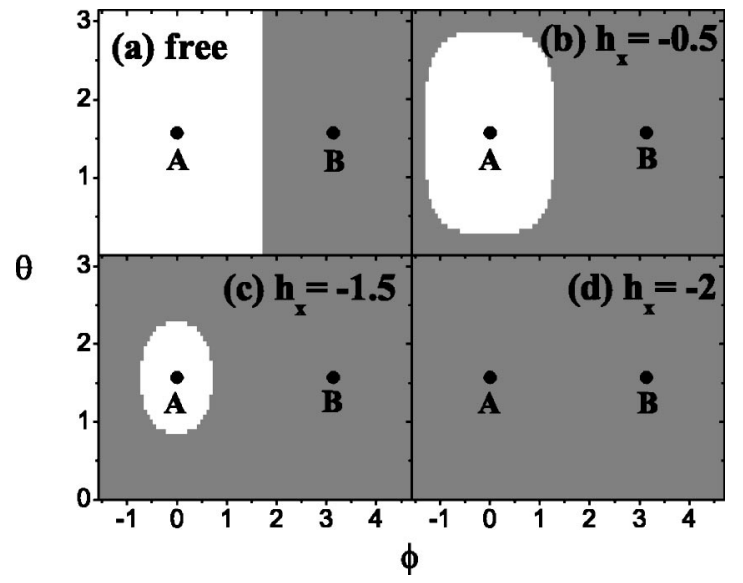

FIG. 6. The basins for $k_{2}=2, k_{4}=0$, and $\alpha=0.1$ with various fields parallel to the easy axis. Points $A$ and $B$, which are also the fixed points, are the initial and target states. The white color is the basin of fixed point $A$ and the grey color is that of $B$. (a) In the absence of an external field, (b) $h_{x}=-0.5$, (c) $h_{x}=-1.5$, and (d), $h_{x}=-2$ (SW limit).

basin is the whole phase plane when the field is above the SW limit.

\section{Field perpendicular to the easy axis}

When a field perpendicular to the easy axis-say, the $z$-direction-is applied, the variations of the fixed points and basins are shown in Fig. 7. The positions of two fixed points shift along the $\theta$ axis, symmetrically located on the two sides of the $+z$ axis. It is evident from Figs. 7(a)-7(c) that the basin shapes become layerlike structures. The outer parts of the basins become layers first. As the field increases, more basin areas become layers. However, the total areas of two basins are equal due to the symmetry of a perpendicular field when the field is below the SW limit. When the field is higher than

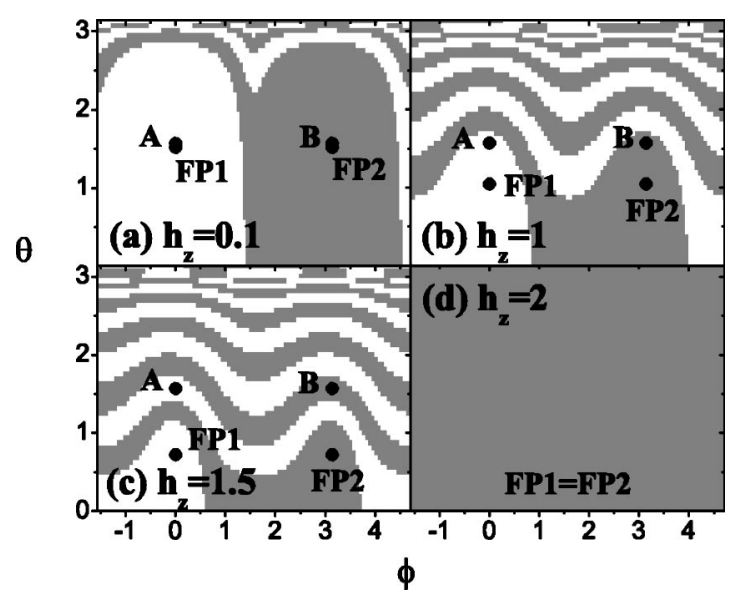

FIG. 7. The basins in the $\theta-\phi$ plane with different strengths of a field perpendicular to the easy axis-say, the $z$ direction. Points $A$ and $B$ denote the initial and target states. The white color is the basin for fixed point FP1 and the grey color is that for FP2. (a) $h_{z}=0.1$, (b) $h_{z}=1$, (c) $h_{z}=1.5$, and (d) $h_{z}=2$ (SW limit). Other parameters are the same as in Fig. 6 .

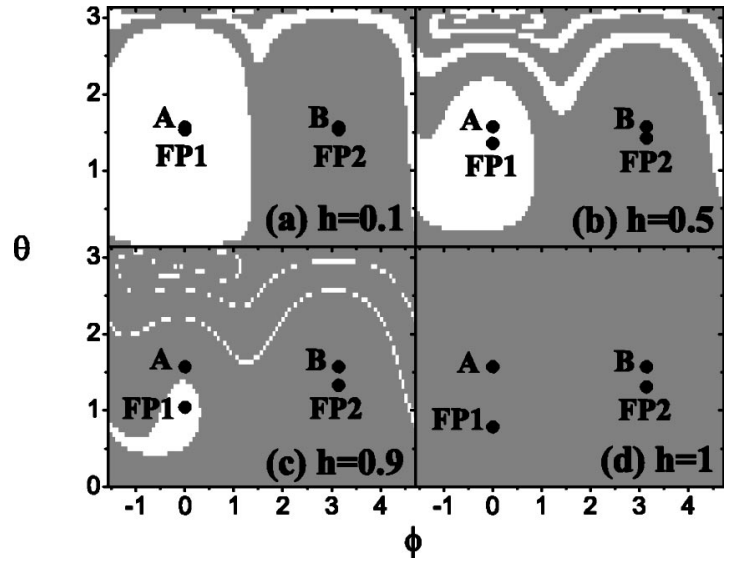

FIG. 8. The basins in the $\theta-\phi$ plane with different $h$ along $135^{\circ}$ to the $+x$ axis. Points $A$ and $B$ denote the initial and target states. The white color is the basin for fixed point FP1 and the grey color is that for FP2. (a) $h=0.1$, (b) $h=0.5$, (c) $h=0.9$, and (d) $h=1$ (SW limit). Other parameters are the same as in Fig. 6. It demonstrates that $A$ and $B$ could be connected by one phase flow trajectory in a field with both nonzero $x$ and $z$ components.

a certain value, point $A$ will fall into the layered region [Fig. 7(c)] and may flow toward the right-hand side of the phase plane. Once the magnetization passes the separatrix line $(\phi=\pi / 2)$, it will evolve into the target state $B$ if one can switch off the field. This precessional procedure was employed in experiments ${ }^{11}$ and numerical calculations. ${ }^{13}$

It is interesting to notice that the widths of these layers become generally thinner when they are away from their cores (central parts of the basins around fixed points) and the number of layers increases with field. The width of a layer is determined by the size of the basin core, energy variation in the phase plane, and energy dissipation during a $360^{\circ}$ precession ( $\phi$ moves from $-\pi / 2$ to $3 \pi / 2$ ). As the field increases, the areas of the core basins shrink, and the precession period is short as well. Thus layers become thinner while the layer number increases. The energy dissipating rate is

$$
\frac{d w}{d t}=-\frac{\alpha}{1+\alpha^{2}}\left|\vec{m} \times \vec{h}_{t}\right|^{2} .
$$

The precession period can be estimated as about $\left(\vec{m} \times \vec{h}_{t}\right)^{-1}$ since $\vec{m} \times \vec{h}_{t}$ is the angular frequency along the latitudes. So the energy loss is proportional to $\left|\vec{m} \times \vec{h}_{t}\right|=\left|\vec{h}_{t} \sin \theta\right|$, which is small for $\theta$ near $\pi$. Thus the width of the outer layers is thinner than that of the inner ones. Figure 7(d) shows that when the field strength reaches the SW limit, the two fixed points merge to the same point $\theta=0$ (north pole).

\section{Field at $135^{\circ}$ to the easy axis}

We now investigate how the basins change under a field with $135^{\circ}$ to the $+x$ direction since the minimal switching field is the smallest around this direction. ${ }^{17}$ Figure 8 is the plot of fixed points and their basins at different field strengths. It has both features of cases with parallel and perpendicular fields. Increasing the field strength, the fixed 
points shall shift along the $\theta$ axis while $\phi$ does not change. Unlike the case of a perpendicular field, the two fixed points have different $\theta$ values. One can see that basin FP1 shrinks while that of FP2 expands as the field strength increases, a feature with parallel field. However, the layer structure also occurs in the outer parts of the basins. The stripes become thinner when the field increases and $\theta$ is away from the fixed point. At the SW limit [Fig. 8(d)], the area of the basin of FP1 shrinks to zero.

\section{Ballistic path}

After seeing the change of fixed points and their basins under different field in the previous section, let us investigate the field and reversal time for the ballistic connection. Without dissipation, the LLG equation is a conservative system. Phase flow is an equipotential curve. As was pointed out in Ref. 19, only a perpendicular field is possible to connect the initial and target states ballistically. Different from the conservative case, ${ }^{19}$ the system starting from $A$ will never pass through the target state $B$ in the presence of dissipation. Even under an infinitely large field, the energy loss during a $180^{\circ}$ precession is not negligible. Although the $180^{\circ}$ precession time $\tau$ decreases as the inverse of the magnetic field, $\tau \sim \pi\left(1+\alpha^{2}\right) / h$ when $h \gg 1$ and $\alpha \ll 1$, the energy dissipation rate goes as

$$
\frac{d w}{d t}=-\frac{\alpha}{1+\alpha^{2}}\left|\vec{m} \times \vec{h}_{t}\right|^{2} \propto h^{2} ;
$$

thus, the energy loss during $\tau$ is proportional to $h$ field. ${ }^{24}$ In order to connect both $A$ and $B$ ballistically, one has to create a small energy difference between $A$ and $B$ such that the energy dissipated on its way from $A$ to $B$ equals the energy difference.

On the other hand, Eq. (3) can be solved exactly in the absence of magnetic anisotropy $\left(k_{2}=0, k_{4}=0\right)$ (Ref. 22) with solution $\phi=h t /\left(1+\alpha^{2}\right)$ and $\cos \theta=\left[\left(1+\cos \theta_{0}\right) e^{2 \alpha h t /\left(1+\alpha^{2}\right)}-1\right.$ $\left.+\cos \theta_{0}\right] /\left[\left(1+\cos \theta_{0}\right) e^{2 \alpha h t /\left(1+\alpha^{2}\right)}+1-\cos \theta_{0}\right]$, where $\theta_{0}$ is the initial angle between the field and magnetization (we assume the field is along the $z$ axis and the initial $\phi$ is zero). Thus ballistic reversal corresponds to applying a field along direction $\theta$ satisfying $-\cos \theta=\left[(1+\cos \theta) e^{2 \alpha \pi}-1+\cos \theta\right] /[(1$ $\left.+\cos \theta) e^{2 \alpha \pi}+1-\cos \theta\right]$. It is interesting to notice that the solution is unique and the angle is $\tan (\theta / 2)=e^{\alpha \pi / 2}$.

Given a damping constant $\alpha$ and magnetic anisotropy, described by $k_{2}$ and $k_{4}$, the $180^{\circ}$ precession time $\tau(h, \beta)$ is a function of field strength $h \equiv \sqrt{h_{x}^{2}+h_{z}^{2}}$ and its angle $\beta$ to the $z$ axis $(\beta$ relates to $\theta$ by $\theta=\pi / 2+\beta)$. Thus the energy dissipated $\Delta \epsilon(h, \beta)=\int_{0}^{\tau}(d w / d t) d t$ during $\tau$ is also a function of $h$ and $\beta$. For $h \gg 1$, one can neglect the magnetic anisotropy and the above isotropic solution should be good. Under the limit, $\Delta \epsilon$ is $2 h\left\{1 /\left[1+\tan ^{2}(\beta / 2+\pi / 4) e^{-2 \alpha \pi}\right]-1 /[1\right.$ $\left.\left.+\tan ^{2}(\beta / 2+\pi / 4)\right]\right\}$. The energy difference $\Delta E(h, \beta)$ between $A$ and $B$ is $2 h \sin (\beta)$. Therefore, a ballistic path must satisfy $\Delta \epsilon=\Delta E$ (a necessary condition but not a sufficient one). Due to symmetric reasons, one needs to consider only $\beta$ $\in(0, \pi / 2)$. Without energy dissipation, the only solution is $\beta=0$ and any $h$ larger than a certain minimal value. With
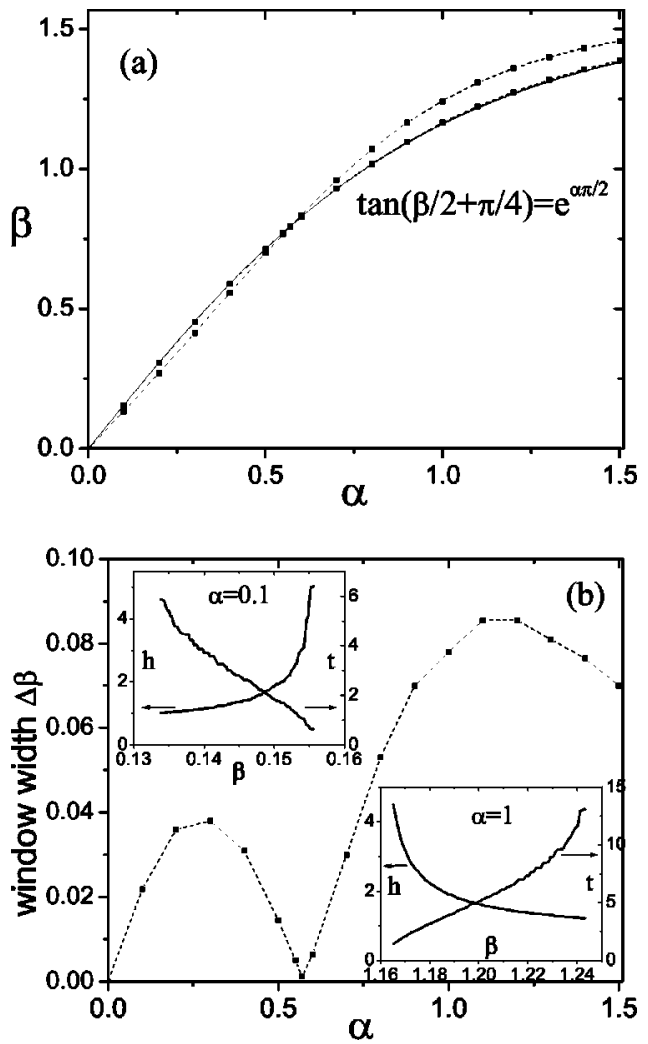

FIG. 9. (a) The upper and lower bounds of $\beta$ as a function of damping constant $\alpha$. The solid line is $\tan (\beta / 2+\pi / 4)=e^{\alpha \pi / 2}$, which should be compared with the upper bound of $\beta$ for $\alpha<0.57$ and the lower bound for $\alpha>0.57$. The magnetic anisotropy is $k_{2}=2$ and $k_{4}=0$. (b) The window width $\Delta \beta$ vs $\alpha$ for $k_{2}=2$ and $k_{4}=0$. Insets: the magnetic field and corresponding reversal time as a function of $\beta$ in the ballistic direction window. $\beta \in(0.134,0.156)$ for $\alpha=0.1$ (upper-left inset), $\beta \in(1.165,1.243)$ for $\alpha=1$ (lower-right inset). The dashed lines are used to guide the eye.

large field $(h \gg 1)$ and energy dissipation $(\alpha \neq 0)$, the approximate solution is $\tan (\beta / 2+\pi / 4)=e^{\alpha \pi / 2}$, the same as the isotropic solution $\tan (\theta / 2)=e^{\alpha \pi / 2}$. For $\alpha \neq 0$ and $k_{2} \neq 0$, we cannot solve $\Delta \epsilon=\Delta E$ analytically. The field configuration of the ballistic connection between $A$ and $B$ was found numerically. The results were displayed in Fig. 9.

Surprisingly, the field can be applied in a range of directions-i.e., a direction window. Given a $\beta$ in this direction window, $h$ is uniquely determined. Both the lower and upper bounds of this $\beta$ window increase with the damping constant. Figure 9(a) is the plot of the upper and lower bounds of $\beta$ as a function of $\alpha$. The solid line is $\tan (\beta / 2+\pi / 4)=e^{\alpha \pi / 2}$. Indeed, the window is around the solid line. The width of the window depends on both the damping constant and magnetic anisotropy. At a zero and infinite damping constant, the width is zero. It is also zero in the absence of magnetic anisotropy as illustrated by the exact solution mentioned in an earlier paragraph. Thus, the width is expected to oscillate with $\alpha$ for a given magnetic anisotropy. This oscillation was indeed observed in numerical calculations as shown in Fig. 9(b) for $k_{2}=2, k_{4}=0$. The upperleft inset of Fig. 9(b) is the field and the corresponding reversal time in the direction window for $\alpha=0.1$ and 
$k_{2}=2, k_{4}=0$. In this particular case, $\beta$ is between 0.134 and 0.156 . One sees that $h$ increases while the reversal time decreases with $\beta$. A similar plot for $\alpha=1$ is shown in the lowerright inset of Fig. 9(b). Opposite to the case of small $\alpha(=0.1), h$ decreases and the reversal time increases with $\beta$. Thus one should compare the lower bound for $\alpha<0.57$ and the upper bound for $\alpha>0.57$ with $\tan (\beta / 2+\pi / 4)=e^{\alpha \pi / 2}$ since it is expected to be exact for $h \rightarrow \infty$ when magnetic anisotropy can be neglected. An excellent agreement was shown in Fig. 9(a). Figure 9(b) is the window width $\Delta \beta$ as a function of $\alpha$. Our numerical results indicate that the perpendicular configuration employed in the current experiments ${ }^{11,12}$ cannot achieve a fully ballistic reversal. It should be pointed out that the above results are for precise ballistic magnetization reversal. As we mentioned earlier, other fields can also switch the magnetization if one will also like to use the ringing effect at certain stages during the reversal process.

\section{E. Discussion}

The SW theory is a classic work ${ }^{10}$ which is based on the energy consideration. Its connection to the LLG theory has not been discussed in the literature as far as we are aware. We clarify that the SW theory is the infinite dissipation limit of the LLG. Under this limit, the magnetization moves along the steepest-energy-descent path. The discrepancy between the SW limit and the numerical minimal switching field has been known for many years. ${ }^{17}$ Also, the existence of a critical value of $\alpha$ has been known numerically. ${ }^{17}$ But its origin was not given in the previous theoretical studies. ${ }^{13,14,17-19}$ To the best of our knowledge, its meaning is first revealed now.

So far, we have reformulated magnetization reversal in terms of the language of nonlinear dynamics. The conventional reversal technique is to make the target state to be the only fixed point while the fast switching method is to connect the initial and target states on the same phase flow trajectory. Dissipation and magnetic anisotropy play very interesting roles in ballistic magnetization reversal. Without dissipation, only perpendicular fields larger than a minimal switching field can create a ballistic path between the initial and target states. In the absence of a magnetic anisotropy and $\alpha \neq 0$, the field direction is not perpendicular but unique, and the field magnitude is arbitrary. In the presence of both dissipation and anisotropy, allowed directions for the ballistic connection form a window. But inside the window, the field magnitude is single-valued. Due to energy dissipation, applying a perpendicular field to the easy axis of a uniaxial Stoner particle cannot directly connect initial and final states by a phase flow trajectory. Thus, the configuration employed in the current fast magnetization reversal experiments ${ }^{11,12}$ is not the best one. A proper field along the direction window can improve the best experimental numbers up to now.
There are advantages and disadvantages for the conventional and fast reversal methods. Although the switching time in the conventional technique is of nanoseconds, the technology is less demanding and easy to implement. On the other hand, the fast switching method could make the switching time at the picosecond scale, but it needs to have precise control of the magnetic pulse. If it is implemented, the cost should be much higher than that of the conventional one. Besides the time issue, the minimal switching field is another concern. The minimal switching field in the conventional reversal technique occurs when only one energy minimum exists in the basin of the final state. Depending on the dissipation rate, the minimal field in the precessional fast switching method may not be much smaller than the SW limit. So far, in both conventional and fast magnetization reversal schemes, both the direction and magnitude of the magnetic field during the pulse duration are assumed to be fixed. If the direction of the magnetic field is allowed to vary with time, the minimal switching field can be even smaller. However, it will be more demanding to implement technologically.

\section{CONCLUSION}

In conclusion, we have shown that magnetization reversal can be conveniently examined in the terminology of nonlinear dynamics. The presence of dissipation will not fail the fast switching method. We clarified that the SW result is the limit of LLG theory with an infinitely large dissipation. In this limit, the magnetization moves along the steepestenergy-descent path. We showed that there is a critical value of the damping constant for a given magnetic anisotropy, above which the minimal switching field is the same as the prediction of SW theory. Based on the change of fixed points and their basins under an external field in different directions, we showed that the magnetization reversal time can be much shorter when the field drives both initial and target states away from fixed points, but puts them on the same phase flow trajectory. In the absence of energy dissipation, the field should be applied perpendicularly to the easy axis in order to achieve a ballistic magnetization reversal. However, with both energy dissipation and a magnetic anisotropy, the field can be applied along a direction window. The width of the window depends on both the damping constant and the magnetic anisotropy. It is zero for either zero damping constant or zero magnetic anisotropy $\left(k_{2}=0\right.$ and $\left.k_{4}=0\right)$. Unlike the conventional magnetization reversal method, our scheme demands precise control of the picosecond pulse of a magnetic field.

\section{ACKNOWLEDGMENTS}

We would like to thank D. Xiao and Q. Niu for sending us their manuscript before publication. This work is supported by UGC, Hong Kong, through RGC CERG grants. 
${ }^{1}$ Robert C. O'handley, Modern Magnetic Materials: Principles and Applications (Wiley, New York, 2000).

${ }^{2}$ Shouheng Sun, C. B. Murray, D. Weller, L. Folks, and A. Moser, Science 287, 1989 (2000).

${ }^{3}$ C. T. Black, C. B. Murray, R. L. Sandstrom, and Shouheng Sun, Science 290, 1131 (2000).

${ }^{4}$ S. I. Woods, J. R. Kirtley, Shouheng Sun, and R. H. Koch, Phys. Rev. Lett. 87, 137205 (2001).

${ }^{5}$ D. Zitoun, M. Respaud, M.-C. Fromen, M. J. Casanove, P. Lecante, C. Amiens, and B. Chaudret, Phys. Rev. Lett. 89, 037203 (2002).

${ }^{6}$ Spin Dynamics in Confined Magnetic Structures I \& II, edited by B. Hillebrands and K. Ounadjela (Springer-Verlag, Berlin, 2001).

${ }^{7}$ W. K. Hiebert, A. Stankiewicz, and M. R. Freeman, Phys. Rev. Lett. 79, 1134 (1997).

${ }^{8}$ Y. Acremann, C. H. Back, M. Buess, O. Portmann, A. Vaterlaus, D. Pescia, and H. Melchior, Science 290, 492 (2000).

${ }^{9}$ T. M. Crawford, T. J. Silva, C. W. Teplin, and C. T. Rogers, Appl. Phys. Lett. 74, 3386 (1999).

${ }^{10}$ E. C. Stoner and E. P. Wohlfarth, Philos. Trans. R. Soc. London, Ser. A 240, 599 (1948), reprinted in IEEE Trans. Magn. 27, 3475 (1991).

${ }^{11}$ C. H. Back, D. Weller, J. Heidmann, D. Mauri, D. Guarisco, E. L. Garwin, and H. C. Siegmann, Phys. Rev. Lett. 81, 3251 (1998); C. H. Back, R. Allenspach, W. Weber, S. S. P. Parkin, D. Weller, E. L. Garwin, and H. C. Siegmann, Science 285, 864 (1999).
${ }^{12}$ H. W. Schumacher, C. Chappert, P. Crozat, R. C. Sousa, P. P. Freitas, J. Miltat, J. Fassbender, and B. Hillebrands, Phys. Rev. Lett. 90, 017201 (2003); H. W. Schumacher, C. Chappert, R. C. Sousa, P. P. Freitas, and J. Miltat, ibid. 90, 017204 (2003).

${ }^{13}$ L. He and W. D. Doyle, J. Appl. Phys. 79, 6489 (1996).

${ }^{14}$ M. Bauer, J. Fassbender, B. Hillebrands, and R. L. Stamps, Phys. Rev. B 61, 3410 (2000).

${ }^{15}$ Y. Acremann, C. H. Back, M. Buess, D. Pescia, and V. Pokrovsky, Appl. Phys. Lett. 79, 2228 (2001).

${ }^{16}$ J. Miltat, G. Albuquerque, and A. Thiaville, in Spin Dynamics in Confined Magnetic Structures I, edited by B. Hillebrands and K. Ounadjela (Springer-Verlag, Berlin, 2001).

${ }^{17}$ L. He, W. D. Doyle, and H. Fujiwara, IEEE Trans. Magn. 30, 4086 (1994).

${ }^{18}$ D. G. Porter, IEEE Trans. Magn. 34, 1663 (1998).

${ }^{19}$ Di Xiao and Qian Niu, cond-mat/0409671 (unpublished).

${ }^{20}$ Textbooks on nonlinear physics, such as S. H. Strogatz, Nonlinear Dynamics and Chaos: With Applications to Physics, Biology, Chemistry, and Engineering (Addison-Wesley, Reading, MA, 1994).

${ }^{21}$ L. Landau and E. Lifshitz, Phys. Z. Sowjetunion 8, 153 (1953); T. L. Gilbert, Phys. Rev. 100, 1243 (1955).

${ }^{22}$ R. Kikuchi, J. Appl. Phys. 27, 1352 (1956).

${ }^{23}$ P. R. Gillette and K. Oshima, J. Appl. Phys. 29, 529 (1958).

${ }^{24}$ One cannot use a small $h$ to switch the magnetization in the presence of a nonzero magnetic anisotropy. A finite $h$ is needed in order to overcome the potential barrier along a reversal path. 